\title{
Clinical analysis of acute coronary syndrome patients with Qi-blood syndromes: establishment of a diagnostic prediction model for syndrome differentiation
}

\author{
Yejing Zhao, Hongyu Peng, Shaoping Wang, Jinghua Liu \\ Department of Cardiology, Beijing Anzhen Hospital, Capital Medical University, Beijing Institute of Heart, Lung and Blood Vessel Diseases, Beijing, \\ China \\ Contributions: (I) Conception and design: All authors; (II) Administrative support: J Liu; (III) Provision of study materials or patients: Y Zhao; (IV) \\ Collection and assembly of data: All authors; (V) Data analysis and interpretation: Y Zhao; (VI) Manuscript writing: All authors; (VII) Final approval \\ of manuscript: All authors. \\ Correspondence to: Jinghua Liu, MD, PhD. Beijing Anzhen Hospital, Capital Medical University, Beijing Institute of Heart, Lung and Blood Vessel \\ Diseases, No. 2 Anzhen Street, Chaoyang District, Beijing 100029, China. Email: liujinghua@vip.sina.com.
}

Background: It has been proven that blood stasis plays an important role in the occurrence and
development of acute coronary syndrome (ACS) due to Qi-stagnation or Qi-deficiency in traditional Chinese
medicine (TCM). However, the diagnosis of Qi-stagnation and blood stasis (QSBS) and Qi-deficiency
and blood stasis (QDBS) syndromes mainly depends on the subjective signs or symptoms in clinical
practice. Using a combination of TCM and modern medicine, this study aimed to investigate the clinical
characteristics of patients with QSBS or QDBS syndromes and to establish a diagnostic prediction model for
Qi-blood syndrome differentiation in clinical practice.
Methods: Patients with ACS who were diagnosed with QSBS syndrome or QDBS syndrome and met
the inclusion criteria were enrolled. Clinical characteristics, laboratory evaluation, and angiographic
characteristics of the two syndrome groups were compared and analyzed. Potential predictive factors
associated with QSBS and QDBS syndromes were explored to establish a diagnostic model for syndrome
differentiation.

Results: A total of 216 participants with ACS, including 108 patients with QSBS syndrome and 108 patients with QDBS syndrome, were enrolled from Beijing Anzhen Hospital Affiliated to Capital Medical University from April 2018 to July 2019. We found that patients with QSBS syndrome were more likely to be males, and have of triple-vessel lesions, relatively high blood stasis syndrome score, normal ejection fraction, and a relatively low-density lipoprotein cholesterol (LDL-C) level. Meanwhile, patients with QDBS syndrome were more likely to have low ejection fraction, high LDL-C level, left main non-triple-vessel lesions, and a relatively low blood stasis syndrome score. The receiver operating characteristic curve and Hosmer-Leme show tests confirmed the discrimination power and reliability of the logistic regression model.

Conclusions: The present study suggested that male sex, the level of LDL-C, ejection fraction, left main lesions, triple-vessel disease, and the score of blood stasis syndrome were the independent predictive factors of Qi-blood syndromes. A good discrimination power of clinical diagnostic prediction model was established using a combination of TCM and modern medicine, and could assist in the differentiation of QSBS syndrome and QDBS syndrome in clinical practice.

Keywords: Traditional Chinese medicine (TCM); Qi-blood syndrome; syndrome differentiation; diagnostic model

Submitted Apr 30, 2020. Accepted for publication Jul 08, 2020.

doi: 10.21037/apm-20-1163

View this article at: http://dx.doi.org/10.21037/apm-20-1163 


\section{Introduction}

Coronary atherosclerotic heart disease (CAD) is one of the major diseases that seriously affects the health of people and incurs large labor and economic losses worldwide (1). Traditional Chinese medicine (TCM), which has a 3,000-year-old history, has a distinct theoretical framework for the diagnosis and treatment of various diseases, including CAD (2). In TCM theory, blood stasis syndrome is considered a pathological state caused by stagnant blood within the body manifesting as various symptoms and signs $(3,4)$. It has been proven that blood stasis plays an important role in the occurrence and development of CAD due to Qi-stagnation or Qi-deficiency. Qi is used to describe the refined nutritious substances constituting the human body and maintaining life activities and the activity of Qi is the driving force of blood circulation. Qi and blood are closely connected, Qi commands blood and blood carries Qi. The dysfunction of Qi and blood is the main pathogenesis of CAD, and further forms different Qi-blood syndromes. Qi-stagnation and blood stasis (QSBS) syndrome and Qideficiency and blood stasis (QDBS) syndrome are the two important subtypes closely related to CAD (5).

The characterization and differentiation of QSBS and QDBS syndromes have played an important role in clinical practice. The treatment in Chinese medicine mainly depends on a dialectical diagnosis, and thus the differentiation of syndrome type can render TCM treatment quite variable. The integrated traditional Chinese and Western medicine has been proved to be safe and effective in the treatment of CAD. In TCM theory, patients with QDBS syndrome should be treated with invigoratingQi and activating-blood formula, while patients with QSBS syndrome should be treated with regulatingQi and activating-blood formula (6). Accordingly, the objectification and standardization of the diagnostic criteria for the differentiation of QSBS and QDBS syndromes would be of great clinical value. However, the diagnosis of QSBS and QDBS syndromes mainly depends on the subjective judgment of symptoms, tongue observation and pulse condition in clinical practice. It is also not yet known whether the objective signs of modern medical technology, such as population characteristics, clinical features, specific biomarkers, molecular mechanism etc., correspond to QSBS and QDBS syndromes in CAD patients. Indeed, because there is a lack of risk factors or indicators for Qi-blood syndromes, QSBS and QDBS syndrome patients cannot be differentiated in an accurate, objective, evidence-based, or quantifiable manner. Therefore, it is necessary to establish a clinical diagnostic prediction model by combining the modern medicine and TCM to assist in the differentiation of QSBS syndrome and QDBS syndrome. The present study was designed to investigate the differences in population characteristics, clinical characteristics, laboratory tests, and coronary lesion features between the two Qiblood syndromes in acute coronary syndrome (ACS) patients, and to screen the predictive factors associated with QSBS and QDBS syndromes. These findings were used to establish a clinical diagnostic prediction model for Qi-blood syndromes in ACS, thus providing a theoretical basis for the objectification of the diagnostic criteria of TCM syndromes in clinical practice and improving the level of accurate diagnosis and precise treatment of Chinese medicine.

We present the following article in accordance with the TRIPOD reporting checklist (available at http://dx.doi. org/10.21037/apm-20-1163).

\section{Methods}

\section{Ethical approval}

The study was conducted in accordance with the Declaration of Helsinki (as revised in 2013), and was approved by the Ethics Committee of Beijing Anzhen Hospital Affiliated to Capital Medical University (no. 2015031). All participants took part in the study voluntarily and signed informed consent.

\section{Study design and participants}

This was a single-center and cross-sectional observational study to identify the objective characteristics of ACS patients with QSBS and QDBS syndromes. A total of 216 participants with ACS, including 108 patients with QSBS syndrome and 108 patients with QDBS syndrome, were enrolled from Beijing Anzhen Hospital Affiliated to Capital Medical University from April 2018 to July 2019. All patients underwent coronary angiography and were scheduled to be treated with percutaneous coronary intervention (PCI). The flow chart for the study design is shown in Figure 1.

\section{Diagnosis criteria}

\section{Diagnostic criteria for ACS}

The diagnostic criteria for ACS were based on the 2013 


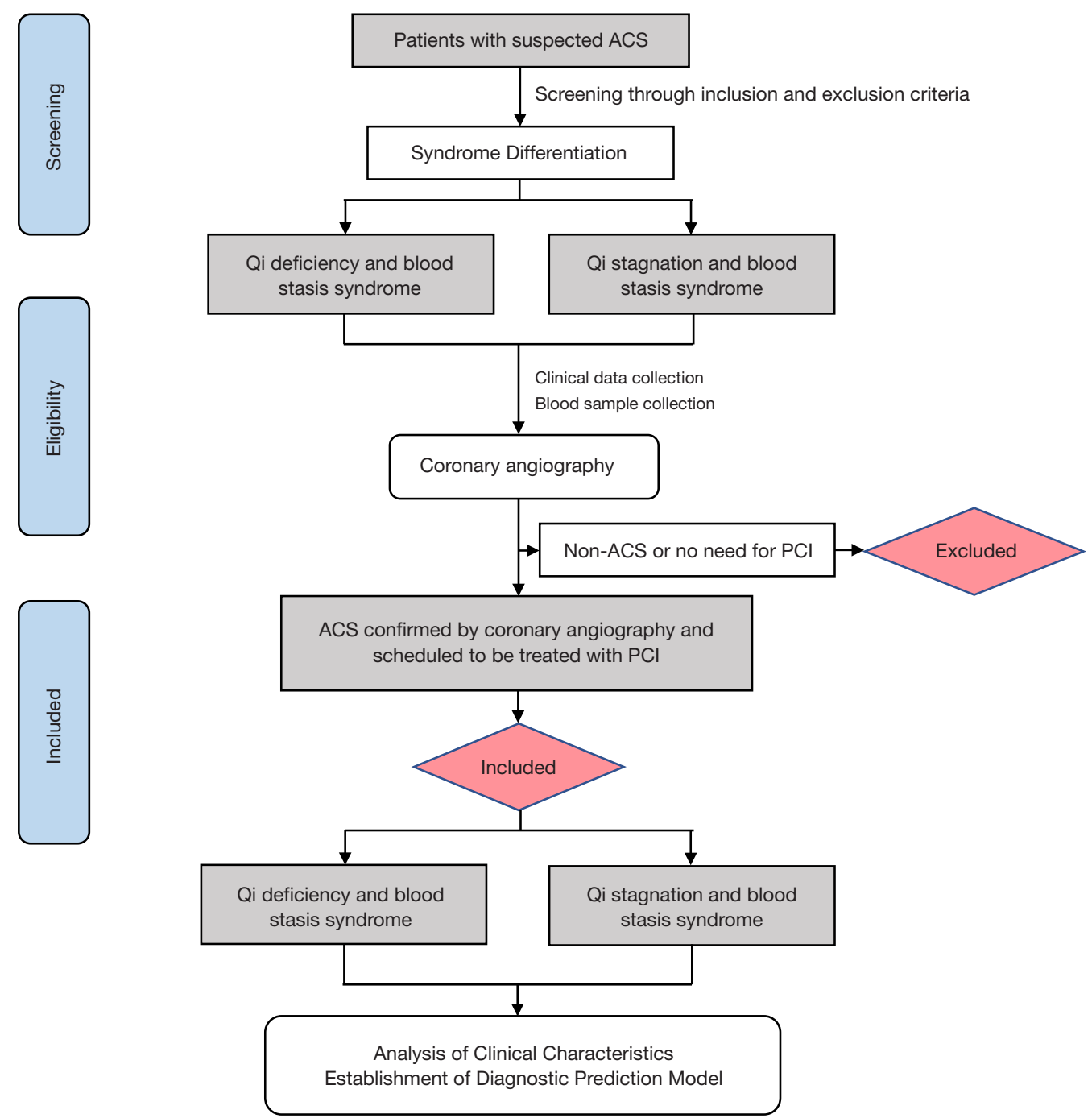

Figure 1 The flow chart of the study design. ACS, acute coronary syndrome; PCI, percutaneous coronary intervention.

American College of Cardiology Foundation and the American Heart Association (ACC/AHA) Guideline for the management of ST-elevation myocardial infarction (MI) (7) and the 2015 European Society of Cardiology (ESC) guidelines for the management of ACS in patients presenting without persistent ST-segment elevation (8).

\section{Diagnostic criteria for syndrome differentiation}

All patients were independently diagnosed by two TCM chief physicians. If the diagnoses were the same, the patient was enrolled. Any disagreements between the two TCM physicians were resolved by discussion with a third investigator. The diagnostic criteria for syndrome differentiation was based on Chinese medicine syndrome differentiation standards of coronary heart disease published by the Professional Committee of Cardiovascular Disease of China Society of Integrated Traditional Chinese and Western Medicine in 1990 (9). The blood stasis syndrome diagnostic scale according to Diagnostic criteria for coronary beart disease patients of blood stasis syndrome was used to assist with the diagnosis and quantitative classification of blood stasis degree (10) (see Table S1).

\section{Inclusion and exclusion criteria}

The inclusion criteria for participants were the following: (I) aged 45-80 years old; (II) diagnosed with ACS; (III) 
with QSBS or QDBS syndrome according to TCM pattern diagnosis; (IV) underwent coronary angiography and required to be treated with PCI; (V) provided voluntary signed informed consent; (VI) full and accurate clinical document and data.

The exclusion criteria for participants were the following: (I) uncontrolled grade 3 hypertension [systolic blood pressure $(\mathrm{SBP})>160 \mathrm{mmHg}$ or diastolic blood pressure $(\mathrm{DBP})>100 \mathrm{mmHg}$ after treatment]; (II) severe cardiopulmonary dysfunction (cardiac function IIIIV, severe abnormal pulmonary function); (III) severe hematopoietic system disorders, liver diseases, renal dysfunction, nervous system diseases, infectious diseases, or malignant tumor; (IV) history of mental illness; (V) pregnant, lactating, or planning pregnancy; (VI) involved in other clinical trials at the same time.

\section{Treatment protocol}

All patients enrolled were grouped by TCM syndrome type within 12 hours after admission. Both the QSBS group and QDBS group received routine medical treatment including dual antiplatelet drugs, beta-blockers, angiotensinconverting enzyme inhibitor (ACEI) or angiotensin II receptor antagonist (ARB), statins, calcium ions antagonists (CCB), nitrates, and proton pump inhibitors (PPIs).

\section{Clinical data collection}

The following information was collected from all participants: gender, height, weight, SBP, DBP, clinical presentation, and previous medical history. Results from electrocardiograms, echocardiography, blood routine tests, blood lipid tests, and blood coagulation tests conducted on each participant before PCI were recorded in detail.

All patients underwent coronary angiography examination in hospital. Quantitative coronary angiography and Gensini score were employed to evaluate the degree of coronary artery stenosis of all participants. Calculation of the Gensini score was performed first by giving a severity score to each coronary stenosis in the following manner: 1 point for $\leq 25 \%$ narrowing, 2 points for $26-50 \%$ narrowing, 4 points for $51-75 \%$ narrowing, 8 points for $76-90 \%$ narrowing, 16 points for $91-99 \%$ narrowing, and 32 points for total occlusion. Thereafter, each lesion score was multiplied by a factor that takes into account the importance of the lesion's position in the coronary circulation ( 5 for the left main coronary artery; 2.5 for the proximal segment of the left anterior descending coronary artery; 2.5 for the proximal segment of the circumflex artery; 1.5 for the midsegment of the left anterior descending coronary artery; 1.0 for the right coronary artery, the distal segment of the left anterior descending coronary artery, the posterolateral artery, and the obtuse marginal artery; and 0.5 for other segments) (11). Finally, the Gensini score was calculated by summation of the individual coronary segment scores.

\section{Blood sample collection}

Fasting venous blood $(5 \mathrm{~mL})$ was collected from all patients in the morning after admission. Blood samples were placed into biochemical tubes and centrifuged for $10 \mathrm{~min}$ at $3,000 \mathrm{r} / \mathrm{min}$ using a refrigerated centrifuge, and then the supernatant was stored in a $-80{ }^{\circ} \mathrm{C}$ refrigerator for the analysis of biological parameters, including the detection of nitric oxide (NO), endothelin-1 (ET-1), ACE, IL-6, TNF- $\alpha$, PLA2G7, ATP, YKL-40, ICAM-1, FGF-21, and CD141.

\section{Determination of biological parameters}

Nitrate reductase method was used to identity the level of NO according to the instruction of matched test kit provided by Nanjing Jiangcheng Bioengineering Institute. ACE concentration was measured by using N-(3-(2-furyl) acryloyl)-Phe-Gly -Gly (FAPGG) as substrate provided by Nanjing Jiangcheng Bioengineering Institute. The levels of ET-1, IL-6, TNF- $\alpha$, PLA2G7, ATP, YKL-40, ICAM-1, FGF-21, and CD141 were determined using enzyme-linked immunosorbent assay (ELISA) methods, and the procedures were performed strictly according to the manufacturer's protocols (R\&D systems, USA).

\section{Statistical analysis}

The sample size estimation by the events per variable (EPV) method indicated that enough subjects were included in the study. Categorical variables were presented as counts and percentages, whereas continuous variables were reported as means and standard deviations (SD) or interquartile range (IQR). The $T$-test was used to assess differences between parametric continuous variables, the MannWhitney $U$ test for non-parametric variables, and the chisquare test or Fisher's exact test were used for categorical variables. Logistic-regression analysis was applied to explore predictive factors associated with QSBS syndrome 
and QDBS syndrome. The model was created with QSBS syndrome being the primary endpoint (QSBS $=1$; QDBS $=0$ ). First, univariate logistic regression analysis was performed to screen out the significant variants from demographic data, medical history, angiographic data, and biological factors $(\alpha=0.10)$. Thereafter, potential variables, on the basis of known clinical relevance or significant association observed at univariate analysis, were entered into the multivariate logistic regression. A binomial multivariate logistic regression model was created using the stepwise method so that only significant variables were included in the final model. Effect estimates were presented as odd ratios (ORs) and $95 \%$ confidence interval (CI). The efficiency of the model as a prediction tool for QSBS and QDBS syndromes in patients with ACS was evaluated by receiver operating characteristic (ROC) curves and Hosmer-Lemeshow test. All tests were two-sided, and a $\mathrm{P}$ value $<0.05$ was considered statistically significant. Calculations were performed using SPSS version 24.0 (IBM Corporation, Chicago, IL, USA).

\section{Results}

\section{Clinical characteristics of patients presenting with QSBS vs. QDBS syndrome}

A total of 216 patients with ACS undergoing PCI were consecutively recruited from Beijing Anzhen Hospital Affiliated to Capital Medical University from April 2018 to July 2019. Among them, 108 patients with QSBS syndrome and 108 patients with QDBS syndrome. The demographic characteristics, laboratory evaluation and angiographic characteristics of all patients enrolled are presented in Tables 1 and 2 .

\section{Demographic characteristics}

Baseline demographic characteristics are summarized in Table 1. The mean ages of the patients enrolled in the QSBS and QDBS groups were both 59 years. The basic clinical characteristics of the two groups, including body mass index (BMI), SBP, DBP, and heart rate, were comparable between the two groups. Most of the patients enrolled in both groups were males; however, there was statistical significance in gender proportion between QSBS and QDBS groups. The male proportion was higher among patients with QSBS syndrome (87/108; 80.6\%), when compared with those with QDBS syndrome (73/108; 67.6\%) ( $\mathrm{P}=0.03)$.

\section{Clinical presentation and medical history}

An admission diagnosis of MI was present in $10.2 \%$ of QDBS syndrome cases and $14.8 \%$ of QDBS syndrome cases, respectively $(\mathrm{P}>0.05)$, and no differences were observed between the two groups regarding previous medical history, such as hyperlipidemia, hypertension, diabetes mellitus, smoking status, previous $\mathrm{MI}$, and previous PCI $(\mathrm{P}>0.05)$.

\section{Medication}

The drug therapy after admission for all patients included aspirin, clopidogrel/ticagrelor and statins. PPI, ACEI, ARB, beta-blockers, nitrates, and CCB were used when necessary, and the frequency of use of these medications did not differ between QSBS and QDBS groups.

\section{Laboratory examination and biochemical factors}

There were no statistical differences in the results of echocardiography and electrocardiogram between the two groups. No differences were observed between the two groups regarding laboratory examination, such as red blood cells, white blood cells, platelet, Alanine transaminase (ALT), Aspartate aminotransferase (AST), Activated Partial Thromboplastin Time (APTT), Prothrombin Time (PT), $\mathrm{D}$-Dimer and so on $(\mathrm{P}>0.05)$. The blood lipids levels, including total cholesterol (TC), triglyceride (TG), and low-density lipoprotein cholesterol (LDL-C), were higher in the QDBS group than in the QSBS group, but this difference was not statistically significant (Table 2).

To identify potential biomarkers of QSBS and QDBS syndrome, biochemical factors associated with endothelial injury and inflammatory response were detected. Among these, the levels of NO, endothelin-1, and PLA2G7 in serum were found to not be significantly different between the two groups $(\mathrm{P}>0.05)$. For the inflammatory mediators, there was a slightly but insignificantly higher level of TNF- $\alpha$, IL-6, and YKL-40 in patients with QSBS syndrome, when compared with those in the QDBS group. The level of ATP, as an energy metabolism factor, was markedly lower in the QDBS group compared with the QSBS group, but not significantly so $(\mathrm{P}>0.05)$ (Table 2$)$.

\section{Angiographic characteristics}

The angiographic characteristics of patients are shown 
Table 1 Comparison of demographic and clinical characteristics between the QDBS group and QSBS group

\begin{tabular}{|c|c|c|c|}
\hline Characteristics & QDBS group $(n=108)$ & QSBS group $(n=108)$ & $P$ value \\
\hline Age, years $($ mean $\pm S D)$ & $59.5 \pm 8.5$ & $58.8 \pm 9.3$ & 0.598 \\
\hline Male, n (\%) & $73(67.6)$ & $87(80.6)$ & 0.030 \\
\hline $\mathrm{BMI}, \mathrm{kg} / \mathrm{m}^{2}($ mean $\pm \mathrm{SD})$ & $25.5 \pm 3.3$ & $25.9 \pm 3.7$ & 0.346 \\
\hline DBP, mmHg (mean \pm SD) & $74.0 \pm 12.4$ & $74.7 \pm 12.2$ & 0.653 \\
\hline Resting heart rate, bpm (mean $\pm \mathrm{SD})$ & $67.8 \pm 8.0$ & $69.4 \pm 10.8$ & 0.231 \\
\hline \multicolumn{4}{|l|}{ Clinical presentation } \\
\hline Diagnosis, n (\%) & & & 0.449 \\
\hline STEMI & $5(4.6)$ & $5(4.6)$ & \\
\hline \multicolumn{4}{|l|}{ Previous medical history, n (\%) } \\
\hline Hypertension & $72(66.7)$ & $72(66.7)$ & 1.000 \\
\hline Hyperlipidemia & $40(37.0)$ & $48(44.4)$ & 0.268 \\
\hline Diabetes mellitus & $36(33.3)$ & $38(35.2)$ & 0.774 \\
\hline Previous smoker & $55(50.9)$ & $53(49.1)$ & 0.785 \\
\hline Previous stroke & $7(6.5)$ & $10(9.3)$ & 0.448 \\
\hline Previous MI & $12(11.1)$ & $13(12.0)$ & 0.832 \\
\hline Ticagrelor & $9(8.3)$ & $11(10.2)$ & \\
\hline Beta blocker & $66(61.6)$ & $57(52.8)$ & 0.216 \\
\hline ACEI/ARA-II & $57(52.8)$ & $61(56.5)$ & 0.585 \\
\hline Statin & $103(95.4)$ & $104(96.3)$ & 0.733 \\
\hline Nitrate & $87(80.6)$ & $85(79.4)$ & 0.838 \\
\hline Calcium antagonist & $32(29.6)$ & $24(22.4)$ & 0.229 \\
\hline Proton-pump inhibitor & $80(74.1)$ & $75(70.1)$ & 0.515 \\
\hline
\end{tabular}


Table 2 Comparison of the laboratory and angiographic characteristics between the QDBS group and QSBS group

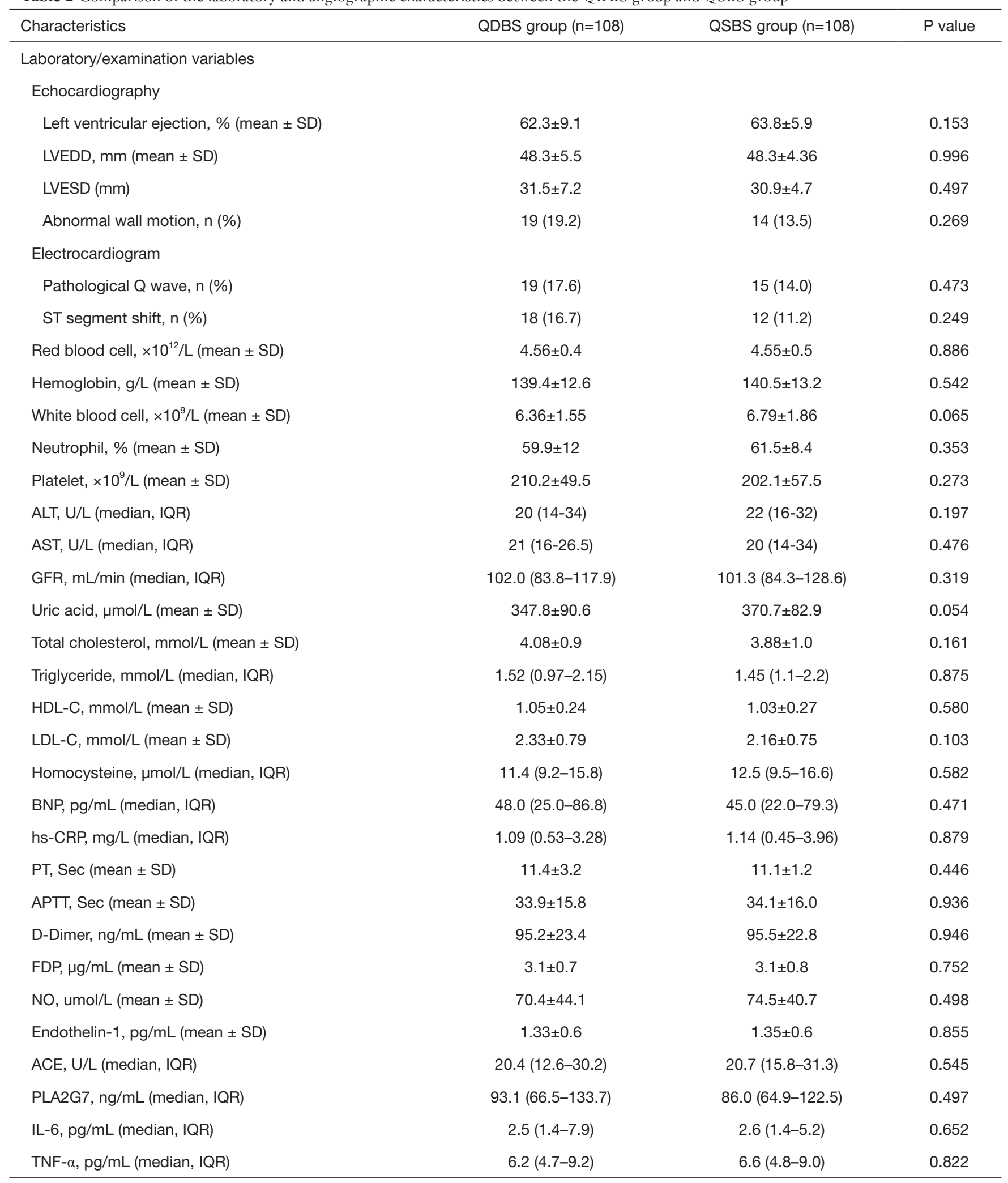

Table 2 (continued) 
Table 2 (continued)

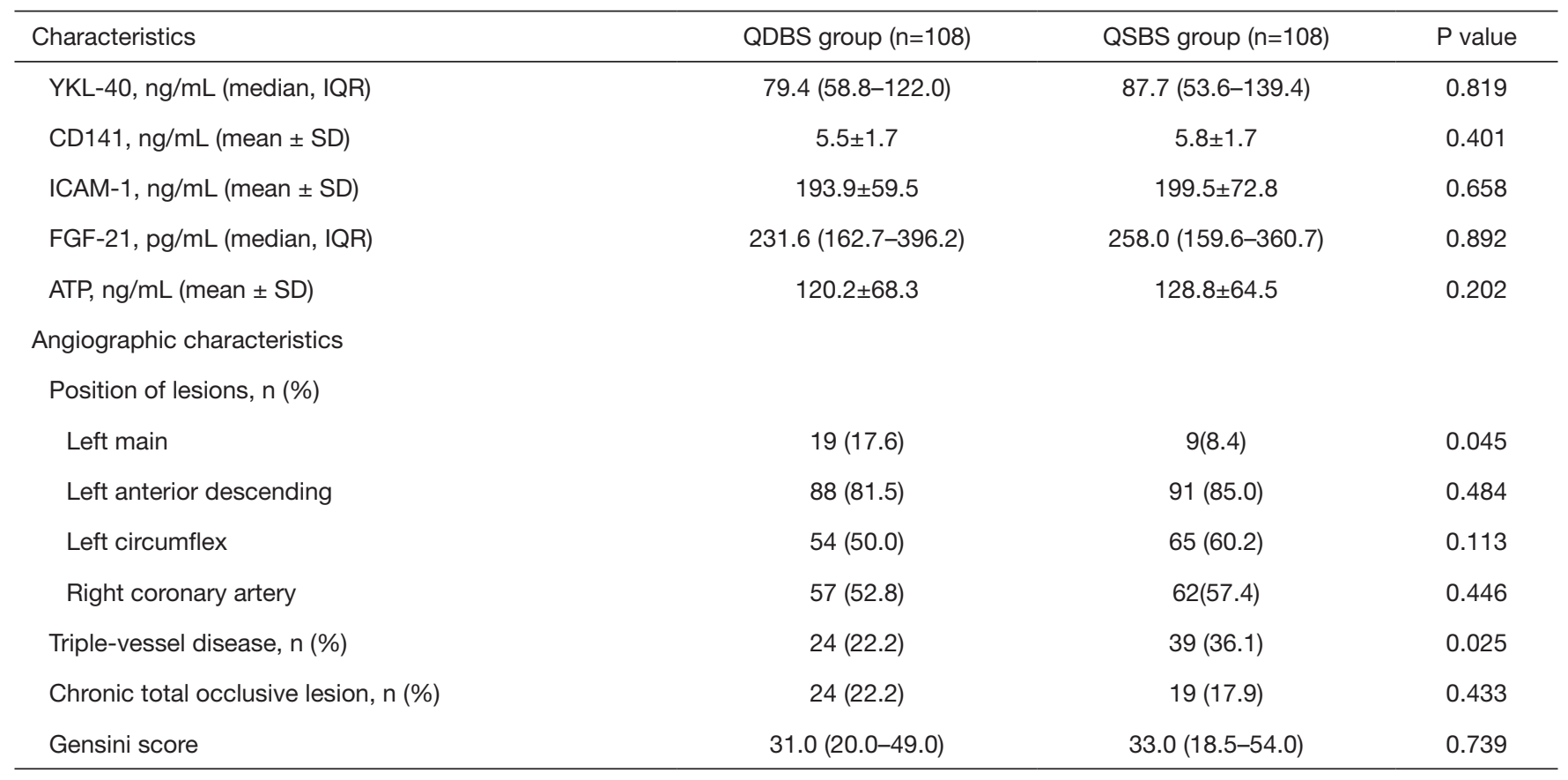

QSBS, Qi-stagnation and blood stasis; QDBS, Qi-deficiency and blood stasis; LVEDD, left ventricular end-diastolic dimension; LVESD, left ventricular end-systolic dimension; ALT, alanine aminotransferase; AST, aspartate aminotransferase; GFR, glomerular filtration rate; HDL-C, high density lipoprotein-cholesterol; LDL-C, low-density lipoprotein-cholesterol; BNP, brain natriuretic peptide; hs-CRP, high sensitive $\mathrm{C}$ reaction protein; $\mathrm{PT}$, prothrombin time; APTT, activated partial thromboplastin time; FDP, fibrinogen degradation product; NO, nitric oxide; SD, standard deviation; IQR, interquartile range.

in Table 2. There was no significant difference in the occurrence of chronic total occlusive lesions and in the Gensini scores of coronary artery stenosis between the QSBS syndrome group and QDBS syndrome group. However, left main coronary lesions appeared more commonly in patients with QDBS syndrome (19/108; $17.6 \%)$ than in patients with QSBS $(9 / 108 ; 8.4 \%)(\mathrm{P}=0.045)$, while the proportion of triple-vessel diseases in patients who were diagnosed with QSBS syndrome was significantly higher than that in patients who diagnosed with QSBS syndrome ( $36.1 \%$ vs. $22.2 \%$; $\mathrm{P}=0.025)$.

\section{The blood stasis syndrome score of patients presenting with QSBS vs. QDBS}

Regardless of syndrome type, all patients had blood stasis. Therefore, the blood stasis degree of each patient was evaluated quantitatively by the Blood Stasis Syndrome Diagnostic Scale published in the Chinese Journal of Integrative Medicine. The blood stasis syndrome score of each patient in both groups is shown in Figure 2 using a scatter plot. The blood stasis score in the QSBS group was higher than that in the QDBS group, indicating that the degree of blood stasis might be relatively more serious in ACS patients with QSBS syndrome.

\section{Logistic regression analysis of predictive factors associated with QSBS and QDBS}

Univariate logistic regression analyses were applied to screen the predictive factors associated with QSBS and QDBS syndromes. It was found that indicators of gender, blood stasis syndrome score, uric acid, LDL-C, left main lesion, and triple-vessel disease were all closely related to the occurrence of Qi-blood syndromes $(\alpha=0.10)$ (Table 3). A binomial multivariate logistic regression model was created using the stepwise method so that only significant variables were included in the final model. The dependent variable was the QSBS/QDBS group; the independent variables were those that were found to be statistically significant in the univariate analysis or had known clinical relevance. In the multivariate analysis, the independent risk factors of 


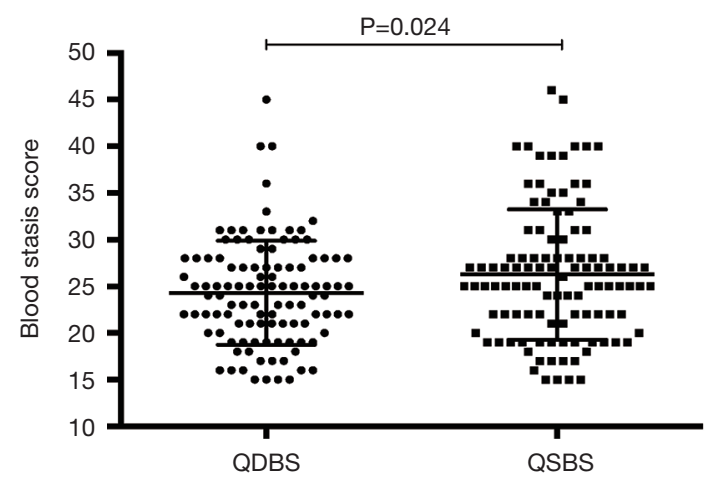

Figure 2 Comparison of the blood stasis syndrome scores between the QDBS syndrome group and the QSBS syndrome group. The blood stasis syndrome scores of patients in the two groups were expressed in the form of scatter plots. QSBS, Qi-stagnation and blood stasis; QDBS, Qi-deficiency and blood stasis.

QSBS syndrome were gender (OR $=2.83$; 95\% CI: 1.39 5.73; $\mathrm{P}=0.004)$, the presence of triple-vessel disease (OR $=2.57 ; 95 \%$ CI: $1.22-5.38 ; \mathrm{P}=0.013)$, and the score of blood stasis syndrome $(\mathrm{OR}=3.77 ; 95 \% \mathrm{CI}$ : $1.01-14.14 ; \mathrm{P}=0.049)$. However, the independent risk factors of QDBS syndrome were the presence of left main lesion $(\mathrm{OR}=2.95 ; 95 \% \mathrm{CI}$ : $1.05-8.30 ; \mathrm{P}=0.040)$, ejection fraction $<50 \%(\mathrm{OR}=4.78$; 95\% CI: $1.03-22.20 ; \mathrm{P}=0.046)$ and LDL-C $>1.8 \mathrm{mmol} / \mathrm{L}$ (OR $=2.20 ; 95 \%$ CI: $1.12-4.33 ; \mathrm{P}=0.022)$. The results of multivariate logistic regression analysis are shown with the forest plots in Figure 3.

\section{Assessment of the diagnostic efficiency of the logistic regression model}

The sensitivity and specificity of the logistic regression model for the diagnoses of QSBS and QDBS syndromes in ACS were assessed using ROC curve analysis. Logistic regression analysis produced an area under the curve (AUC) of 0.734, as shown in Figure 4, with non-significant Hosmer-Lemeshow tests confirming the reliability of the model.

\section{Discussion}

According to TCM theory, Qi and blood are the two most important substances and energy foundations of the human body. The physiological functions of the body mainly rely on the circulation of blood and Qi (12). It has been proven that dysfunction of Qi and blood is the main pathogenesis of CAD, and further forms different Qi-blood syndromes. QDBS syndrome and QSBS syndrome are the two important subtypes closely related to CAD. Essentially, the two sub-syndromes are both caused by the disorder of Qi that eventually lead to the occurrence of blood stasis. However, there are difference between the two syndromes. Qi-stagnation means that the movement of Qi is blocked, which leads to the occurrence of blood stasis; Qi-deficiency means that insufficient generation or excessive consumption of Qi, which leads to the occurrence of blood stasis. Therefore, it also underlines the difference in treatment between QDBS syndrome and QSBS syndrome. Patients with QDBS syndrome could benefit from invigoratingQi and activating-blood therapy, however, patients with QSBS syndrome should be treated with regulating-Qi and activating-blood therapy. Qi-blood theory has been widely applied in the diagnosis and treatment for CAD. However, objective prediction factors that could assist in the diagnosis and differentiation of QDBS syndrome and QSBS syndrome still do not exist. The current methods of syndrome differentiation are mostly based on personal experience, and there is a lack of precision and objectivity in the diagnosis of Qi-blood syndromes, which greatly hinder the development of the Qi-blood theory and the clinical popularization of TCM. Therefore, how to develop the Qi-blood theory of syndrome differentiation, achieve accurate diagnoses, and broaden clinical application have remained the most persistent barriers for TCM in recent years. Epidemiological observation of population characteristics, exploration of the predictive factors, and establishment of objective diagnostic models are important ways to achieve the accuracy and standardization of Qi-blood syndrome differentiation.

In the present study, 216 ACS patients with QDBS or QSBS syndrome were enrolled from April 2018 to July 2019. The objective characteristics of the two groups, including demographic and clinical characteristics, laboratory evaluation, and angiographic characteristics, were compared and analyzed. Potential predictive factors associated with QSBS and QDBS syndromes were explored to establish a diagnostic model for Qi-blood syndrome differentiation. The principal finding in the current study was that Qi-blood syndrome differentiation was closely associated with gender, low-density lipoprotein, ejection fraction, left main lesion, triple-vessel disease, and blood stasis syndrome score. More specifically, male sex, triplevessel disease, and a higher blood stasis syndrome score were independent risk factors of QSBS syndrome, while the presence of left main lesion, low ejection fraction, and 
Table 3 Logistic regression models for QSBS/QDBS syndromes

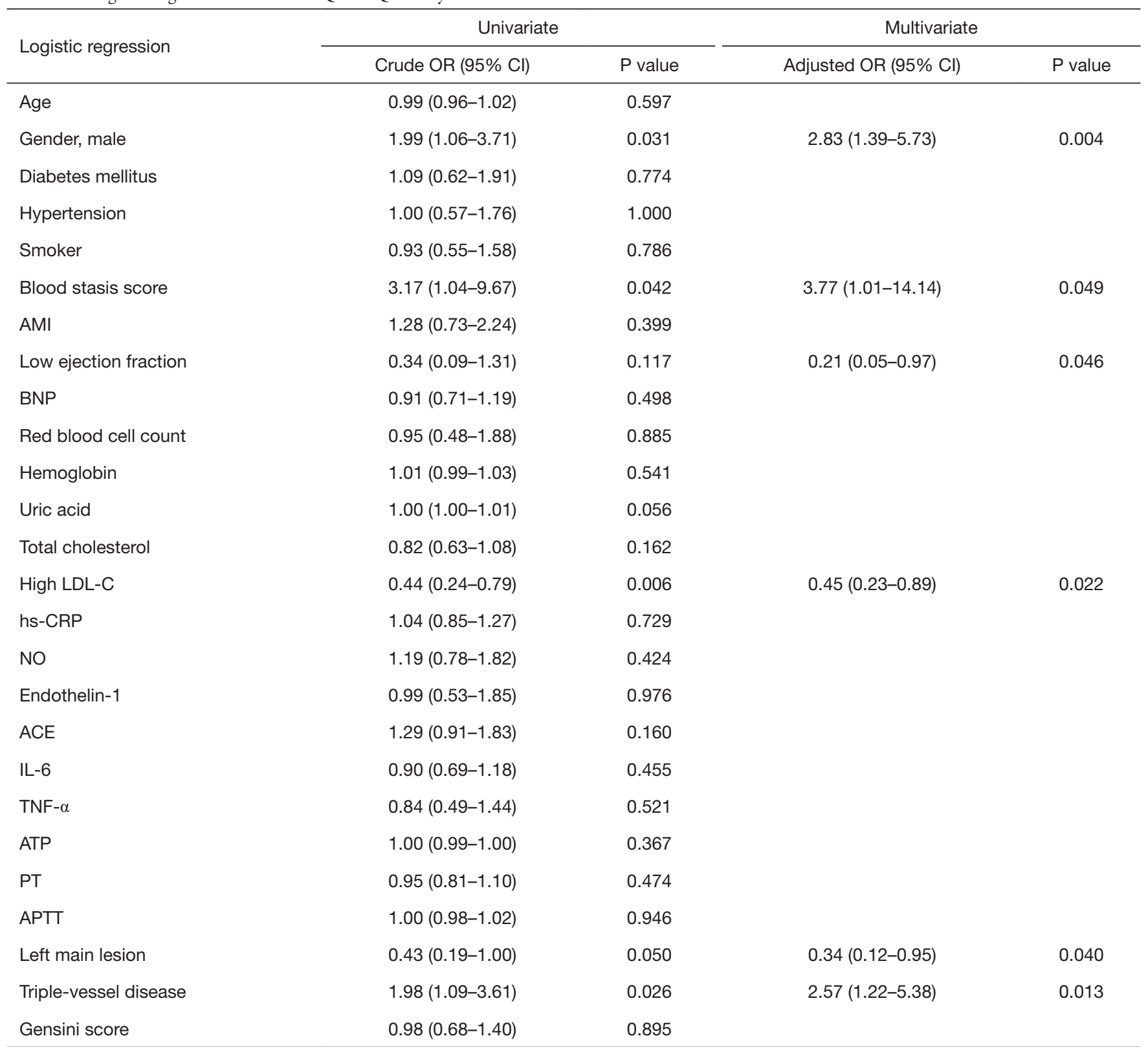

The logistic regression model was created with QSBS syndrome being the primary endpoint (QSBS =1; QDBS =0). QSBS, Qi-stagnation and blood stasis; QDBS, Qi-deficiency and blood stasis; AMI, acute myocardial infarction; BNP, brain natriuretic peptide; LDL-C, low-density lipoprotein-cholesterol; hs-CRP, high sensitive C reaction protein; NO, nitric oxide; PT, prothrombin time; APTT, activated partial thromboplastin time; OR, odds ratio; $\mathrm{Cl}$, confidence interval.

high LDL-C were the independent risk factors of QDBS syndrome.

\section{Qi-blood syndrome differentiation and gender}

The present study revealed that gender difference had a certain impact on the distribution of Qi-blood syndromes among patients with ACS. The proportion of male in patients with QSBS syndrome was relatively higher compared with the patients diagnosed with QDBS syndrome, and male sex was confirmed to be an independent predictive factor of Qiblood syndrome according to regression analysis. This result 


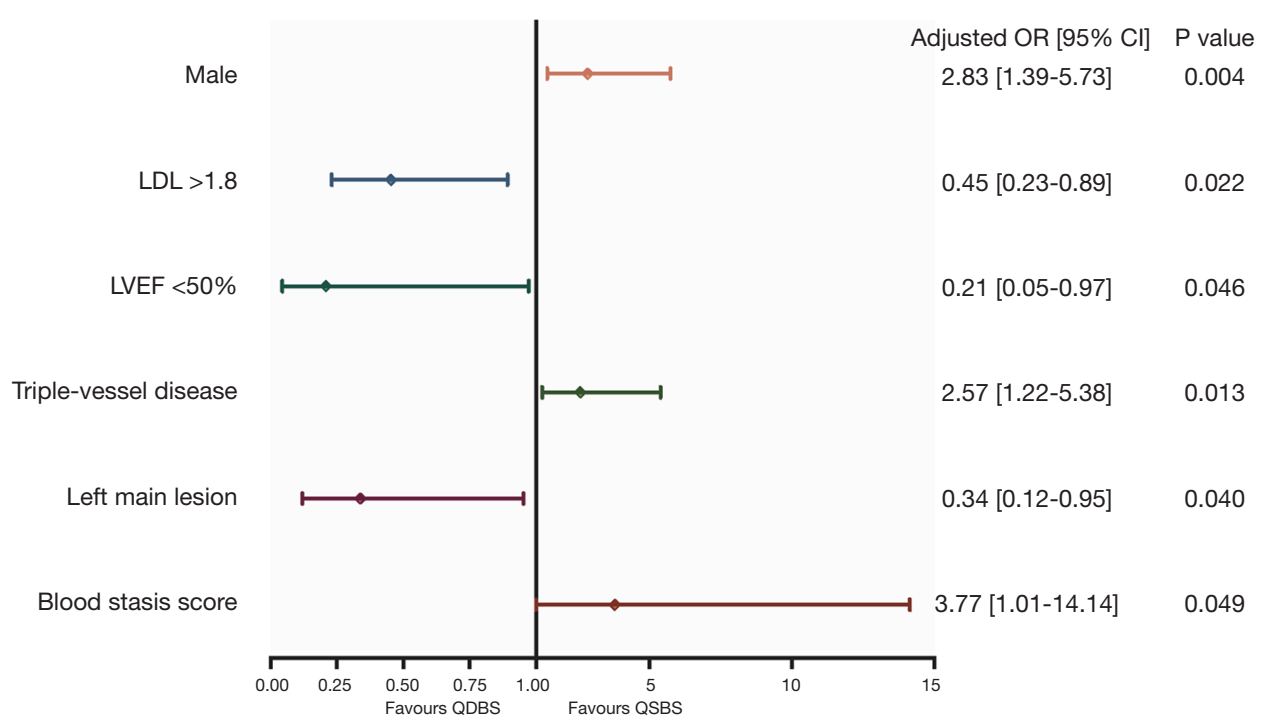

Figure 3 The forest plots for multivariate logistic regression analysis. QSBS, Qi-stagnation and blood stasis; QDBS, Qi-deficiency and blood stasis; LDL, low-density lipoprotein; LVEF, left ventricular ejection fraction; OR, odds ratio.

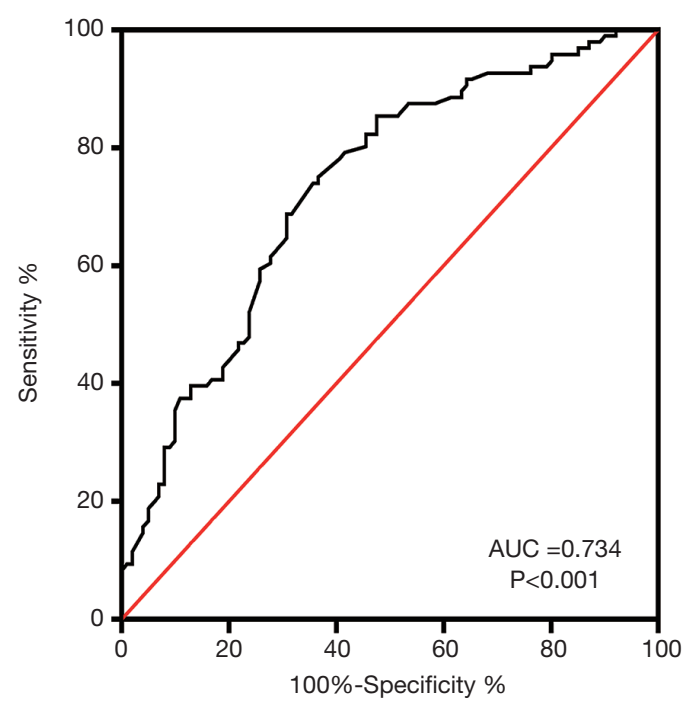

Figure 4 ROC curves for the multivariate logistic model. ROC, receiver operating characteristic.

is consistent with the previous studies. Wen et al. have also found the influence of gender difference on the syndrome differentiation of excess and deficiency. The manifestations of the syndrome in males were mostly excess syndromes, such as blood stasis and Qi-stagnation. However, females were more likely to show deficiency syndromes, such as Qi-deficiency and Yin deficiency. The influence of gender differences on the distribution of Qi-blood syndromes may be related to the different physiological structures of men and women (13). The result of our study also further hinted at the differences in TCM treatment between men and women. Guided by syndrome differentiation of excess and deficiency, it is worth noting that male patients with ACS may require more attention in regulating Qi and activating blood therapy, while female patients with ACS may be more likely to benefit from invigorating-Qi and activating-blood therapy.

\section{Qi-blood syndrome differentiation and lipid metabolism}

Dyslipidemia, categorized as "phlegm turbidity", has been proven to be the main risk factor of blood stasis syndrome in TCM. Previous studies have found that dyslipidemia has a certain correlation with TCM syndrome classification in patients with CAD. A study by Ren et al. (14), which included 23 patients with QSBS syndrome and 55 patients with QDBS syndrome, showed that the levels of TC and TG in patients with QDBS syndrome were increased when compared with the levels in patients with QSBS syndrome. Our study indicated that there was no significant statistical difference in blood lipid levels between QDBS and QSBS groups, but there was a trend of increase in the levels of TC, TG, and LDL-C in patients with QDBS syndrome when compared with patients with QSBS syndrome. Furthermore, the present study confirmed that high 
LDL-C (>1.8 mmol/L) was an independent risk factor of QDBS syndrome. To a certain extent, the results suggest that the efficiency of substance metabolism of patients with QDBS syndrome may decrease and cause fat metabolism disorder. This is consistent with the viewpoint of TCM theory which holds that Qi-deficiency cannot transmit or transform nutrients of "grain" and "water", which leads to disorders in the metabolism of materials in the human body.

\section{Qi-blood syndrome differentiation and cardiac ejection fraction}

Previous research on TCM has demonstrated that Qideficiency may be the important initiating factor of heart failure (15-17). As ancient physicians remark, "Qi is the sovereign of the blood: normal flow of Qi ensures normal flow of the blood, while deficiency of Qi causes stagnation of the blood". Several studies found the cardiac function of patients with CAD of QDBS syndrome to be obviously changed, as characterized by a decrease of cardiac output, stroke output, and cardiac index when compared with healthy controls (18-20). From this point of view, the present study was devoted to exploring whether cardiac function could be used as an independent predictive factor for ACS patients with QDBS syndrome, and had the aim of providing valuable information for the differential diagnosis of Qi-blood syndromes. Our synthesis provides overwhelming evidence that the decrease of ejection fraction (EF $<50 \%$ ) is one of the independent predictive factors for differentiating between QDBS syndrome from QSBS syndrome in patients with ACS. In modern medicine, a series of mechanisms, including impaired myocardial energy metabolism, weakened myocardial systolic and diastolic function, and impaired ejection capacity of the heart, have been associated with the occurrence of heart failure (21). According to the theory of TCM, the heart governs the blood, and the heart-Qi of patients with QDBS syndrome is deficient that could cause stagnation of blood. Therefore, the heart-Qi of patients with QDBS syndrome is deficient if it is unable to push the blood forward and leads to the decrease of cardiac ejection fraction. Our results further confirmed the important role of Qi in the occurrence and development of this disease.

\section{Qi-blood syndrome differentiation and angiographic characteristics}

Numerous studies have been devoted to exploring the correlation between the angiographic characteristics and distribution of TCM syndromes. Previous studies have shown that the severity of coronary artery lesions in patients with blood stasis syndrome is significantly more serious than that in patients without blood stasis syndrome $(22,23)$. However, the characteristics of coronary angiography between QDBS syndrome and QSBS syndrome in patients with $\mathrm{CAD}$ remains controversial. Some studies hold the view that QDBS syndrome is closely related to mild coronary stenosis and that QSBS syndrome is closely related to multivessel coronary artery diseases (24-27), while others believe the opposite $(28,29)$. In the present study, our findings showed that there was no significant difference in Gensini score between the two groups; however, triple-vessel disease was an independent risk factor for QSBS syndrome, while the presence of left main lesion and non-triplevessel disease were the independent risk factors for QDBS syndrome. It can be seen that different researchers have failed to draw a consistent conclusion for the characteristics of coronary angiography between QDBS syndrome and QSBS syndrome. One of the reasons for this is that different dialectical theories of TCM syndrome differentiation have been adopted by different researchers. For instance, our study was mainly based on the theory of Qi-blood, and did not focus on Yang deficiency, Yin deficiency, phlegm turbidity, and other syndromes. Moreover, the dialectical diagnosis of TCM is relatively subjective and is closely related to the doctor's personal experience. Meanwhile the subjects selected for each study were different: some studies enrolled patients with unstable angina pectoris $(25,26)$, some enrolled patients with ACS (27), while others enrolled only patients with $\operatorname{CAD}(24,28,29)$, and this may have some effect on the results. Therefore, large multicenter clinical studies are warranted to further explore the issue.

\section{Qi-blood syndrome differentiation and blood stasis syndrome score}

Both Qi-stagnation and Qi-deficiency can eventually lead to the outcome of blood stasis syndrome in patients with CAD. Chinese medicine holds that blood stasis plays an important role in the occurrence and development of CAD (5). In the present study, the Blood Stasis Syndrome Scale was used to quantitatively evaluate the degree of blood stasis status in patients. It was found that the blood stasis syndrome scores of patients with QSBS syndrome were significantly higher than those of patients with QDBS syndrome, and higher score of blood stasis syndrome was confirmed to be an independent 
risk factor of QSBS syndrome. The results also suggest that the degree of blood stasis in patients with ACS and the QSBS subtype, who were more likely to have triple-vessel lesions, may be relatively more serious. Therefore, intensive treatment of improving blood circulation and dispersing stasis in these patients seems particularly important.

\section{Qi-blood syndrome differentiation and biochemical factors}

Links between inflammation and cytokines have been proven to play important roles in the development of atherosclerotic cardiovascular disease (30). Previous studies have explored the relationship between the TCM dialectical types of CAD and inflammatory factors or cytokines. Hong et al.'s study found that the TCM syndromes of CAD were correlated with inflammatory factors, such as CRP, IL-6, and TNF-a, with these inflammatory factors being significantly increased in ACS patients with blood stasis syndrome (31). A study by Qi et al. has shown that the Qi-deficiency syndrome of unstable angina pectoris was related to inflammatory factors, including IL, hs-CRP, FIB, TNF-a, and ICAM-1. The inflammation level of patients with Qi-deficiency syndrome is higher than that of healthy people but lower than that of non-Qi deficiency syndrome patients (32). Ren et al.'s study detected the level of inflammatory mediators in the serum of subjects with Qi-blood dysfunction and found that the levels of TNF- $\alpha$ and LN in the serum of patients with QDBS syndrome and QSBS syndrome were significantly higher, while the level of slgA was lower when compared with those of the healthy control group (14). Overall, these previous studies indicate that inflammatory reaction may exist in the process of congestion caused by Qi disease in CAD. Our study compared the level of related biochemical factors in the serum of patients in the QDBS group and QSBS group and found that the levels of inflammatory factors and vasoactive substances in the QSBS group, including hs-CRP, IL6 , TNF- $\alpha$, YKL-40, ICAM-1, and CD141, were higher compared with those in the QDBS group. However, there was no statistical difference. This suggests that patients with QSBS syndrome may have a stronger inflammatory reaction and hypercoagulability in the development and progression of ACS. However, our results still need to be further confirmed by metabonomics and genomics testing.

\section{Establishment of a clinical diagnostic prediction model}

The diagnosis of QSBS and QDBS syndromes mainly depends on the subjective judgment and clinical experience of doctors, while the diagnostic criteria still lack support from objective indicators of modern medical technology. To the best of our knowledge, this is the first study reported that establishes a clinical diagnostic prediction model by analyzing the clinical characteristics, laboratory examinations, and coronary angiographic features of patients with QSBS syndrome and QDBS syndrome. We found that patients with QSBS syndrome were more likely to be males, and have three-vessel lesions, relatively high blood stasis syndrome score, normal ejection fraction, and relatively low LDL-C level. Meanwhile, patients with QDBS syndrome were more likely to have low ejection fraction, high LDL-C level, left main and non-threevessel lesions, and relatively low blood stasis syndrome score. The ROC analysis and the Hosmer-Lemeshow test both indicated the good predictive adjustment and discriminative power of the regression model. The establishment of a model in this study may provide a theoretical support for increasing the objectivity of the diagnostic criteria of QSBS and QDBS syndrome in clinical practice.

\section{Limitations}

The main limitation of our study is that the efficacy of the clinical diagnostic prediction model still needs to be improved and validated, and these works will be completed in further research. Biomarkers with significant differential diagnostic value for Qi-blood syndromes were not found in our research. Therefore, further large-scale, multicenter clinical trials along with metabonomics and genomics testing are needed to identify biomarkers with higher diagnostic value, and apply them to the establishment of a diagnostic model. This way, a better tool with higher diagnostic efficiency can be developed.

\section{Conclusions}

Patients with QSBS syndrome were more likely to be males, and have three-vessel lesions, relatively high blood stasis syndrome score, normal ejection fraction, and a relatively low LDL-C level; meanwhile, patients with QDBS syndrome were more likely to have low ejection fraction, high LDL-C level, left main and non-threevessel lesions, and a relatively low blood stasis syndrome score. By combining modern medicine and TCM, were able to achieve good discrimination power in a clinical 
diagnostic prediction model that may have clinical value in differentiating between QSBS syndrome and QDBS syndrome. Further large-scale, multicenter clinical trials, along with metabonomics and genomics research, are warranted to refine more precise and objective tools with higher discriminative power, thus improving the accuracy of diagnosis and treatment in modern Chinese medicine.

\section{Acknowledgments}

Funding: This study was supported by the National Basic Research Program of China (973 programs, No. 2015CB554404).

\section{Footnote}

Reporting Checklist: The authors have completed the TRIPOD reporting checklist. Available at http://dx.doi. org/10.21037/apm-20-1163

Data Sharing Statement: Available at http://dx.doi. org/10.21037/apm-20-1163

Conflicts of Interest: All authors have completed the ICMJE uniform disclosure form (available at http://dx.doi. org/10.21037/apm-20-1163). The authors have no conflicts of interest to declare.

Ethical Statement: The authors are accountable for all aspects of the work in ensuring that questions related to the accuracy or integrity of any part of the work are appropriately investigated and resolved. The study was conducted in accordance with the Declaration of Helsinki (as revised in 2013), and was approved by the Ethics Committee of Beijing Anzhen Hospital Affiliated to Capital Medical University (No. 2015031). All participants took part in the study voluntarily and signed informed consent.

Open Access Statement: This is an Open Access article distributed in accordance with the Creative Commons Attribution-NonCommercial-NoDerivs 4.0 International License (CC BY-NC-ND 4.0), which permits the noncommercial replication and distribution of the article with the strict proviso that no changes or edits are made and the original work is properly cited (including links to both the formal publication through the relevant DOI and the license). See: https://creativecommons.org/licenses/by-nc-nd/4.0/.

\section{References}

1. Kosmas CE, Silverio D, Sourlas A, et al. Antiinflammatory therapy for cardiovascular disease. Ann Transl Med 2019;7:147.

2. Wang C, Niimi M, Watanabe T, et al. Treatment of atherosclerosis by traditional Chinese medicine: Questions and quandaries. Atherosclerosis 2018;277:136-44.

3. Park B, You S, Jung J, et al. Korean studies on blood stasis: an overview. Evid Based Complement Alternat Med 2015;2015:316872.

4. You S, Park B, Lee MS. Accelerated RBC senescence as a novel pathologic mechanism of blood stasis syndrome in traditional East Asian medicine. Am J Transl Res 2015;7:422-9.

5. Jiang $\mathrm{M}$, Zhang $\mathrm{C}$, Cao $\mathrm{H}$, et al. The role of Chinese medicine in the treatment of chronic diseases in China. Planta Med 2011;77:873-81.

6. Jiang M, Lu C, Zhang C, et al. Syndrome differentiation in modern research of traditional Chinese medicine. J Ethnopharmacol 2012;140:634-42.

7. Levine GN, Bates ER, Bittl JA, et al. 2016 ACC/ AHA Guideline Focused Update on Duration of Dual Antiplatelet Therapy in Patients With Coronary Artery Disease: A Report of the American College of Cardiology/ American Heart Association Task Force on Clinical Practice Guidelines: An Update of the 2011 ACCF/AHA/ SCAI Guideline for Percutaneous Coronary Intervention, 2011 ACCF/AHA Guideline for Coronary Artery Bypass Graft Surgery, 2012 ACC/AHA/ACP/AATS/PCNA/ SCAI/STS Guideline for the Diagnosis and Management of Patients With Stable Ischemic Heart Disease, 2013 ACCF/AHA Guideline for the Management of STElevation Myocardial Infarction, 2014 AHA/ACC Guideline for the Management of Patients With NonST-Elevation Acute Coronary Syndromes, and 2014 ACC/AHA Guideline on Perioperative Cardiovascular Evaluation and Management of Patients Undergoing Noncardiac Surgery. Circulation 2016;134:e123-55.

8. Roffi M, Patrono C, Collet JP, et al. 2015 ESC Guidelines for the Management of Acute Coronary Syndromes in Patients Presenting Without Persistent ST-segment Elevation. Rev Esp Cardiol (Engl Ed) 2015;68:1125.

9. Chinese Medicine Syndrome Differentiation Standards of Coronary Heart Disease. Chin J Integr Med 1991;6:257.

10. Diagnostic Criteria for Coronary Heart Disease Patients of Blood Stasis Syndrome. Chin J Integr Med 1987;7:129-31.

11. Gensini GG. A more meaningful scoring system for 
determining the severity of coronary heart disease. Am J Cardiol 1983;51:606.

12. Yao W, Yang H, Ding G. Mechanisms of Qi-blood circulation and Qi deficiency syndrome in view of blood and interstitial fluid circulation. J Tradit Chin Med 2013;33:538-44.

13. Weng C, Cheng W. A Study on the Relationship between Information of 206 Patients with Angina Pectoris and TCM Syndrome Differentiation. Hubei Journal of Traditional Chinese Medicine 2002;24:3-4.

14. Ren JX, Liu JX, Lin CR. Comparative analysis on the biological basis of blood stasis syndrome induced by qistagnation and qi-deficiency in patients with unstable angina pectoris. Zhongguo Zhong Xi Yi Jie He Za Zhi 2010;30:352-6.

15. Li XQ, He JC, Huang PX, et al. Chinese medicine syndromes in congestive heart failure: A literature study and retrospective analysis of clinical cases. Chin J Integr Med 2016;22:738-44.

16. Wang Q, Yao GZ, Pan GM, et al. Analysis of on medication rules for Qi-deficiency and blood-stasis syndrome of chronic heart failure based on data mining technology. Zhongguo Zhong Yao Za Zhi 2017;42:182-6.

17. Bai D, Yue GX, Wang RH, et al. Clinical characteristics of five traditional Chinese medicine injections in treating heart failure based on Meta-analysis literature. Zhongguo Zhong Yao Za Zhi 2018;43:4152-62.

18. Liu J, Dong W. Preliminary study on pathophysiological characteristics of patients with qi stagnation and blood stasis and Qi deficiency and blood stasis. J Tradit Chin Med 1991:46-7.

19. Zhang Y, Huang X, Li Y. Comparison of Hemorheology and Cardiac Function of Qi Stagnation and Blood Stasis Syndrome, Qi Deficiency and Blood Stasis Syndrome in Coronary Heart Disease. Liaoning TCM Magazine 1996;09:10-11.

20. Ding L. A Clinical Study on the Relationship between TCM Syndrome Differentiation and Left Ventricular Function in Coronary Heart Disease. Forum On Traditional Chinese Medicine 2018;33:32-3.

21. Yancy CW, Jessup M, Bozkurt B, et al. 2017 ACC/AHA/ HFSA Focused Update of the 2013 ACCF/AHA Guideline for the Management of Heart Failure: A Report of the American College of Cardiology/American Heart Association Task Force on Clinical Practice Guidelines and the Heart Failure Society of America. J Card Fail 2017;23:628-51.

22. Zhou X, Liang H, Sun X. Meta-analysis of correlation between TCM blood stasis syndrome and coronary angiography. Chinese Journal of Evidence-Based Medicine 2012;12:1470-7.

23. Liu H, Wang Z, Shang J. A study on the correlation between coronary angiography and blood stasis syndrome in patients with coronary heart diseases. Journal of Cardiovascular and Cerebrovascular Diseases with Integrated Traditional Chinese and Western Medicine 2007;03:189-90.

24. Wang J, He QY, Ma CS. Research on relationship between coronary lesion and blood stasis syndrome based on coronary angiography. Zhongguo Zhong Xi Yi Jie He Za Zhi 2008;28:1074-7.

25. Wang Y. Study on the correlation between TCM syndromes of unstable angina pectoris and Gensini score of coronary artery and plasma atherosclerotic index. Fujian University of Traditional Chinese Medicine 2019.

26. Deng D, Zhao H, Chen J. Study on the correlation between Gensini score of coronary artery and TCM syndromes in patients with unstable angina pectoris of coronary heart disease. Chinese Medical Emergency 2016;25:1649-51.

27. Zhu C, Yan K, Zhu M. Study on the correlation between TCM syndromes of coronary heart disease and coronary artery disease. China J Chin Mater Med 2009;34:3289.

28. Shi L, Liu H, Wang Z. Study on the correlation between coronary Gensini score and TCM syndromes of patients with coronary heart disease. World Journal of Integrated Traditional Chinese and Western Medicine 2008;3:722-4.

29. Zhang P, Liu P, Xu W. 368 cases of coronary artery disease characteristics and TCM syndrome type correlation study. Journal of Cardiovascular and Cerebrovascular Diseases with Integrated Traditional Chinese and Western Medicine 2010;08:1308-11.

30. Buffon A, Biasucci LM, Liuzzo G, et al. Widespread coronary inflammation in unstable angina. N Engl J Med 2002;347:5-12.

31. Hong Y, Huang Y, Wu H, et al. Clinical Study on the Relationship between TCM Syndromes of Coronary Heart Disease and Inflammatory Factors. Journal of Guangzhou University of Traditional Chinese Medicine 2005;22:81-6.

32. Qi Q, Li Y, Chen J, et al. Study on the correlation between Qi deficiency syndrome and inflammation in unstable angina pectoris of coronary heart disease. Journal of Cardiovascular and Cerebrovascular Diseases with Integrated Traditional Chinese and Western Medicine 2010;08:1418-20.

Cite this article as: Zhao Y, Peng H, Wang S, Liu J. Clinical analysis of acute coronary syndrome patients with Qi-blood syndromes: establishment of a diagnostic prediction model for Qi syndrome differentiation. Ann Palliat Med 2020;9(4):20962110. doi: 10.21037/apm-20-1163 
Supplementary

Table S1 The quantitative evaluation table for blood stasis syndrome of coronary heart disease

\begin{tabular}{|c|c|}
\hline Item & Score \\
\hline No & 0 \\
\hline $\begin{array}{l}\text { Mild: typical angina attacks, lasting several minutes, at least 2-3 times a week, but the pain is not serious, sometimes } \\
\text { nitroglycerin is needed }\end{array}$ & 3 \\
\hline $\begin{array}{l}\text { Extremely severe: typical angina pectoris, severe degree, frequent attacks, duration }>30 \text { minutes, unable to be alleviated by } \\
\text { nitroglycerin, or clinical diagnosis of acute myocardial infarction }\end{array}$ & 15 \\
\hline \multicolumn{2}{|l|}{ Tongue nature } \\
\hline Medium: dark red tongue, scattered red dots or ecchymosis & 6 \\
\hline Severe: purple tongue & 9 \\
\hline \multicolumn{2}{|l|}{ Lips } \\
\hline No & 0 \\
\hline Mild: dark red lips & 3 \\
\hline Medium: dark lips & 6 \\
\hline Severe: dark lips, gums can be seen red dots or ecchymosis & 9 \\
\hline \multicolumn{2}{|l|}{ Varicose sublingual vein } \\
\hline No & 0 \\
\hline Lusterless pulse & 8 \\
\hline Uneven pulse & 10 \\
\hline Total score & \\
\hline
\end{tabular}

